\title{
COMPARISON OF SURFACE CHARACTERISTICS OF THE ANTARCTIC ICE SHEET WITH SATELLITE OBSERVATIONS \\ (Abstract)
}

by

\author{
N. Young and I. Goodwin
}

(Antarctic Division, Department of Science, Kingston, Tasmania 7150, Australia)

\begin{abstract}
Ground surveys of the ice sheet in Wilkes Land, Antarctica, have been made on oversnow traverses operating out of Casey. Data collected include surface elevation, accumulation rate, snow temperature, and physical characteristics of the snow cover. By the nature of the surveys, the data are mostly restricted to line profiles. In some regions, aerial surveys of surface topography have been made over a grid network.

Satellite imagery and remote sensing are two means of extrapolating the results from measurements along lines to an areal presentation. They are also the only source of data over large areas of the continent. Landsat images in the visible and near infra-red wavelengths clearly depict many of the large- and small-scale features of the surface. The
\end{abstract}

intensity of the reflected radiation varies with the aspect and magnitude of the surface slope to reveal the surface topography. The multi-channel nature of the Landsat data is exploited to distinguish between different surface types through their different spectral signatures, e.g. bare ice, glaze, snow, etc. Additional information on surface type can be gained at a coarser scale from other satellite-borne sensors such as ESMR, SMMR, etc. Textural enhancement of the Landsat images reveals the surface micro-relief.

Features in the enhanced images are compared to ground-truth data from the traverse surveys to produce a classification of surface types across the images and to determine the magnitude of the surface topography and micro-relief observed. The images can then be used to monitor changes over time. 